\title{
Compliance with Postoperative Recommendations by Patients with Different Psychological Types after Early Implant Loading Treatment
}

\author{
Bogdan Pelekhan *iD, Mykola Rozhko, Lyubomyr Pelekhan, Olena Rozhko
}

\begin{abstract}
The objective of the research was to explore the relationship between different psychological types of patients with an edentulous mandible and their attitudes toward postoperative recommendations and prescriptions after early implant loading treatment.

Materials and Methods. The study was conducted at the Dentistry Center (University Clinic, Ivano-Frankivsk National Medical University), November 2019 - March 2020. Study group consisted of 28 individuals with an edentulous mandible. Patients were divided into subgroups (philosophical, exacting-mind, indifferent, and hysterical) based on their mental characteristics. Surveys about complaints, compliance with postoperative recommendations and prescriptions were performed on the $2^{\text {nd }}, 4^{\text {th }}, 8-10^{\text {th }}$ and $12-14^{\text {th }}$ days after surgical treatment.

Results. Clinical evaluation data, patients' complaints, and compliance with the postoperative treatment protocol after early implant loading treatment were analyzed. The degree of compliance to prescribed recommendations in patients with different psychological types were evaluated and discussed.

Conclusions. According to the results within the limitations of this study, it can be concluded that compliance with postoperative recommendations and prescriptions is highly dependent on the patient' psychological type.
\end{abstract}

\section{Keywords}

Edentulism; Psychological Type; Implant Treatment; Mental Classification; Postoperative Treatment

The Department of Dentistry of Postgraduate Study Faculty, Ivano-Frankivsk National Medical University, Ivano-Frankivsk, Ukraine *Corresponding author: bpelechan@gmail.com

\section{Problem statement and analysis of the latest research}

Edentulism is a widespread problem that varies from country to country, depends on socioeconomic and age-dependent factors, as well as dental care and oral health knowledge factors [1] and has important impact on a patient's general health and life quality $[2,3]$. Currently, implant treatment plan of edentulous patients is widely used and shows the most acceptable long-term outcomes $[4,5]$. Successful implant treatment of edentulous patients depends on different risk factors, type of pre- and postoperative management, complications in the early period [6]. The patients' oral health knowledge, their attitude toward postoperative recommendations [7-9] and understanding the importance of prescribed pharmacological protocol have a significant impact as well [10]. The level of patients' attitude often depends on communication and dentist's explanation of successful implant treatment [11]. However, patient mental type has a direct influence on dental surgeon-patient collaboration and outcomes of edentulism treatment [12]. At the same time, the dentist's understanding of the patient's psychological type is helpful in choosing better communication and cooperation tactics to achieve successful results of implant treatment and reduce complications in the early postoperative period.

The objective of the research was to explore the relationship between different psychological types of patients with an edentulous mandible and their attitudes toward postoperative recommendations and prescriptions after early implant loading treatment. 


\section{Materials and Methods}

\section{Study Group}

This descriptive study was conducted at the Dentistry Center (University Clinic, Ivano-Frankivsk National Medical University), November 2019 - March 2020. Study group included 28 patients with an edentulous mandible (10 males and 18 females; the mean age - 51.7 [min - 42; max - 75] years).

The indications and contraindications for implant treatment were analyzed and the possibility of the chosen treatment plan was evaluated. Medical history taking, consultations and examinations were conducted by general practitioners before the surgical stage. Patients without concomitant pathology were included in the study.

All the patients were divided into four groups (Table 1) according to the M.M. House mental classification [13].

\section{Dental Implant Treatment of the Edentulous Mandible}

The surgical stage of dental implant treatment consisted of installing intraosseous dental implants and healing abutments with soft tissues suturing using monofilament suture material capable of resorption to $50 \%$ of strength in 8-13 days and to $0 \%$ in $90-120$ days.

\section{Postoperative Treatment Plan and Clinical Evalua- tion}

Patients were prescribed medical treatment according to the pharmacological protocol (Appendix 1) and postoperative recommendations were provided (Appendix 2) after the surgical stage of dental implantation.

Postoperative clinical evaluation was performed on the $2^{\text {nd }}$, $4^{\text {th }}, 8-10^{\text {th }}, 12-14^{\text {th }}$ days according to early implant loading and dental prosthesis manufacturing protocols. It included the patients' general well-being, analysis of complaints, evaluation of peri-implant soft tissues.

The data about the patients' complaints, compliance with the pharmacological protocol and recommendations were collected using designed standard questionnaire (Appendix 3). The patients' answers were categorized as full/partial/no compliance.

Oral hygiene's level was determined by the Winkel Tongue Coating Index (WTCI) [14] on the $8^{\text {th }}-10^{\text {th }}$ days of the postoperative period. It was based on the evaluation of different tongue coating regions (Fig. 1) and performed according to Lundgren $\mathrm{T}$ et al. [15]. Score 0 was assigned for region with no coating; score 1 was assigned for region with a light thin layer; score 2 was assigned for region with a heavy thick layer. Thus, the WTCI of each patient was obtained by summing the score for each region (WTCI $=\mathrm{A}+\mathrm{B}+\mathrm{C}+\mathrm{D}+\mathrm{E}+\mathrm{F}$ ).

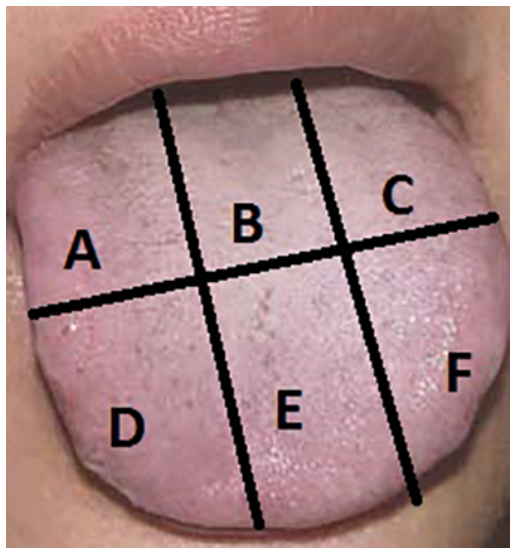

Figure 1. Nominal division of the tongue for WTCI evaluation.

\section{Data Analysis}

Data processing (WTCI calculation, description of survey results as percentage and frequency) and graphical representation were performed in MS Excel 2016.

\section{Results}

\section{Examination on the $2^{\text {nd }}$ Day}

The patients of Group 1 (75\% of individuals) complained of unpleasant achy pain in the oral cavity only. Typical complaints in Group 2 were bleeding sensations and pink saliva (66.7\% of patients), trouble sleeping (55.6\% of patients), pain (77.8\% of patients). The most common complaint in Group 3 (66.7\% of patients) was the dental implant pain.

A common complaint in patients of Group 4 (hysterical psychological type) was pain characterized as unbearable, stabbing, and uncontrolled. Due to this complaint, $20 \%$ of patients were ready to have their implants removed.

The results of the survey showed a high level of compliance with the prescribed pharmacological protocol and recommendations (Fig. 2) by patients of Group 1 (88.8\%), Group 2 $(100 \%)$ and Group $4(80 \%)$. However, $83.3 \%$ of patients of Group 3 followed the protocol partially, without specifying

Table 1. Groups of patients according to mental classification.

\begin{tabular}{cllcc}
\hline Group & Psychological type & Characteristics & $\mathrm{n}$ & $\%$ \\
\hline 1 & philosophical & sensible, calm, motivated, and ready for long-term treatment & 8 & 28.8 \\
2 & exacting-mind & insistence on high standards, personal organization, and accurateness & 9 & 32.1 \\
3 & indifferent & apathetic, disinterested, and low-emotional in communication & 6 & 21.4 \\
4 & hysterical & emotional instability, irritability, and imbalance & 5 & 17.7 \\
\hline Total & & & 28 & 100 \\
\hline
\end{tabular}



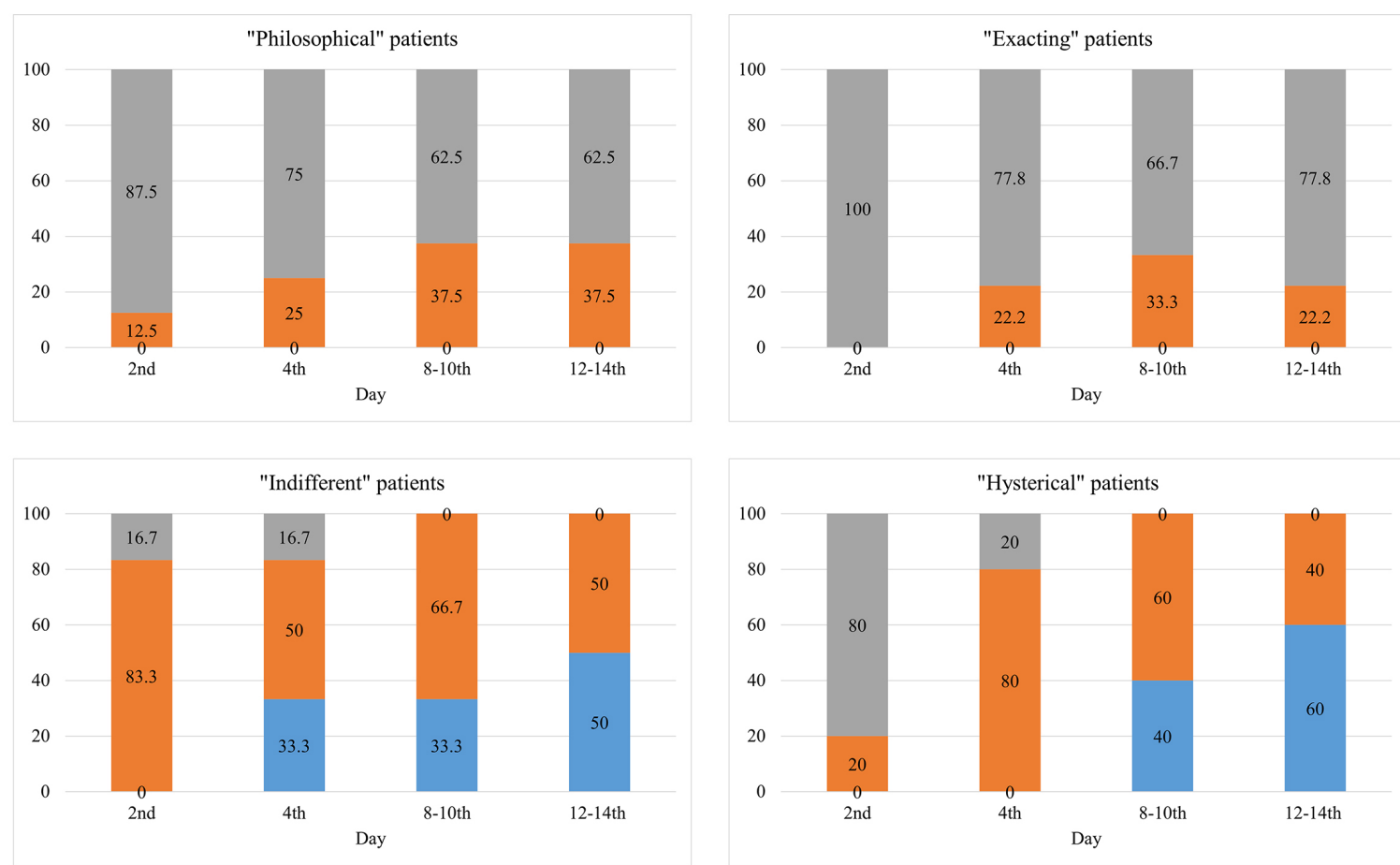

no compliance $\square$ partial compliance $\square$ full compliance

Figure 2. Patients' compliance with the prescribed pharmacological protocol and recommendations.

the reason. Clinical examination of patients in Group 3 determined poor oral hygiene level, food debris, halitosis; edema of the maxillofacial area was more extensive than in patients of other groups.

\section{Examination on the $4^{\text {th }}$ Day}

The total number of complaints decreased in patients of all groups on the $4^{\text {th }}$ day. Group 1 patients indicated a decrease in pain dynamics. Group 2 patients showed particular concern about the presence of postoperative edema which, however, tended to decrease. Patients of Group 3 and Group 4 developed new complaints of discomfort associated with suture material.

A common feature of all groups was a decrease in compliance with the prescribed pharmacological protocol and recommendations (Fig. 2). However, the most significant decrease was noted in Group 3.

\section{Examination on the $8^{\text {th }}-10^{\text {th }}$ Days}

Patients' complaints and general tendency of compliance with the pharmacological protocol and recommendations (Fig. 2) were preserved in Group 1 and Group 2 with a slight decrease in Group 3 and Group 4, as evidenced by the WTCI (Fig. 3).

According to the results of the WTCI (Fig. 3), oral hygiene was poor in patients of Group 3 and Group 4 (indifferent and hysterical types, respectively); satisfactory level was observed

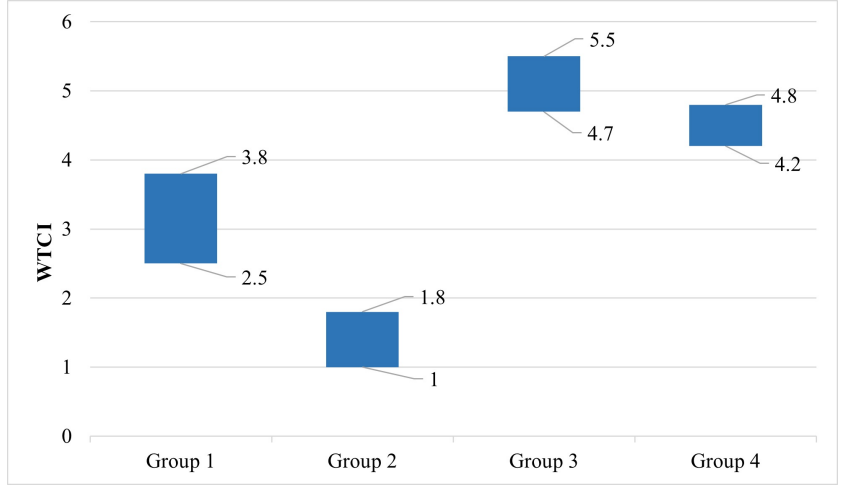

Figure 3. WTCI in patients of all groups.

in patients of Group 1 (philosophical type); the highest level was found in patients of Group 2 (exacting-mind type).

\section{Examination on the $12^{\text {th }}-14^{\text {th }}$ Days}

Patients' complaints were not significant; however, adhering to the pharmacological protocol and recommendations decreased in Group 3 and Group 4 (Fig. 2). Clinical examination showed high dependence between compliance with postoperative recommendations and peri-implant tissue healing. Tissue condition in 100\% of patients in Group 1 and Group 2 (Fig. 4, 5) allowed for suture removal and prosthetic restoration fixation. However, in $83.3 \%$ and $60 \%$ of Group 3 
and Group 4 patients (Fig. 6, 7), respectively, these manipulations had to be postponed with the subsequent control of the peri-implant tissues in 2 days.

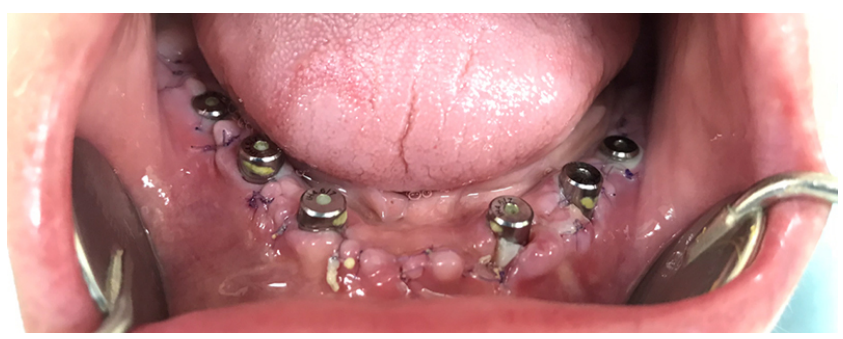

Figure 4. Peri-implant tissues in a patient of Group 1 (philosophical type) on the $12^{\text {th }}$ day.

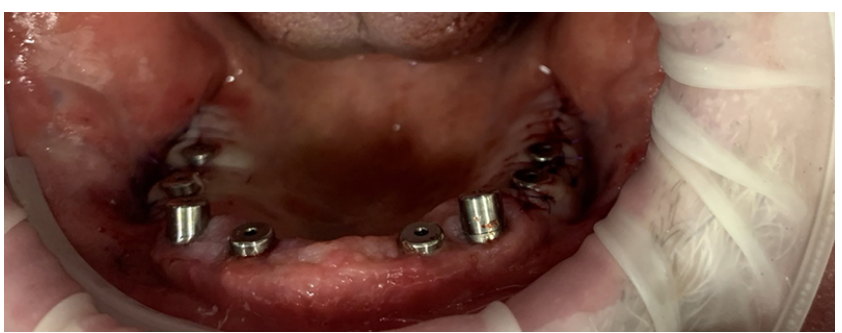

Figure 5. Peri-implant tissues in a patient of Group 2 (exacting-mind type) on the $12^{\text {th }}$ day.

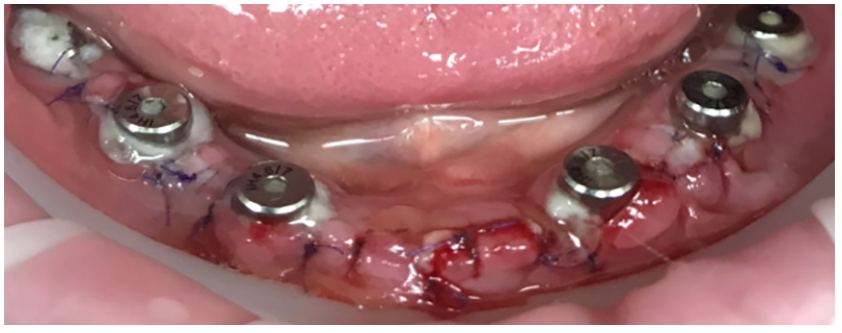

Figure 6. Peri-implant tissues in a patient of Group 3 (indifferent type) on the $12^{\text {th }}$ day.

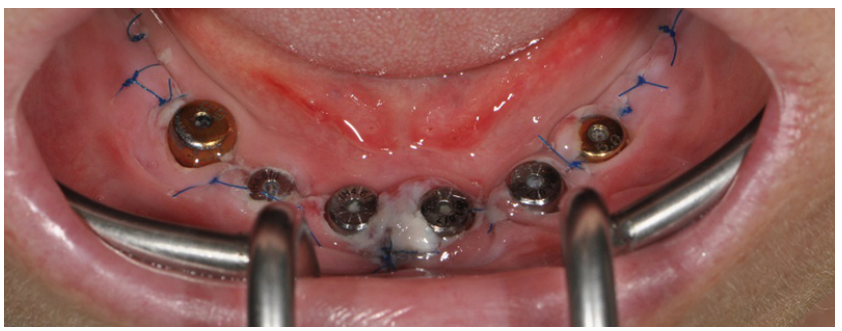

Figure 7. Peri-implant tissues in a patient of Group 4 (hysterical type) on the $12^{\text {th }}$ day.

\section{Discussion}

The global prevalence of edentulism continues to be high [16] However, dental health care is focused on decreasing edentulism rate via increasing oral hygiene knowledge, preventive dental care, and treatment. It includes an increase in the efficacy of implant prosthodontic treatment. Successful implant treatment of edentulism is highly dependent on peri-implant tissues and oral hygiene level $[17,18]$.

Implant treatment planning is multicomponent and should include understanding patients' psychology [19]. According to our study, patients varied in their levels of submision to prescribed recommendations. The most ideal dental patients were characterized by philosophical and exacting-mind types. Their emotional sphere was stable, they demonstrated a high level of readiness to treatment, followed recommendations in more than $62.5 \%$ of cases. These points were in line with the study conducted by Gamer S et al. [19]. Hysterical patients were emotionally unstable, demonstrated strong agreement with dentists' recommendations at the beginning of observation which decreased dramatically over rehabilitation time. At the same time, additional verbal support and motivation strategy did not lead to a positive effect in all cases $(60 \%$ of patients did not demonstrate compliance with recommendations). This was consistent with the study carried out by Monje A et al. [20] which showed that changing the communication strategy did not always have a strong positive impact on improving patient's motivation and response. Both indifferent and hysterical patients did not demonstrate any comprehension of postoperative treatment importance. These patients followed the prescriptions and/or recommendations partially or did not comply with the prescriptions and/or recommendations, without any reason.

Psychological background plays an important role in patients' understanding biological aspects of their disease and dental care $[21,22]$. Our results demonstrated high dependence between the patients' complaints and their psychological type. Philosophical and exacting-mind individuals demonstrated an understanding of complaints. Motivating communication and explanation that pain and other complaints were physiological had the positive effect, as evidenced by the decrease in pain complaint on the $4^{\text {th }}$ day of the postoperative period. However, additional verbal communication with hysterical and indifferent patients did not have significant satisfactory effect on their attitude toward complaints.

The level of oral hygiene is an important factor of successful treatment. Food debris, plaque accumulation, and marginal bone loss have been reported to be interrelated and have influence on implant stability rate [23-25]. We found philosophical and exacting-mind patients to have significantly higher oral hygiene level (low values of the WTCI); however, indifferent and hysterical patients demonstrated opposite results which may lead to dissatisfactory outcomes in long-term observation. This confirmed the fact that such patients required additional motivation and explanation through the improvement of communication skills and dentist-patient 
collaboration [26].

This study had a number of limitations. Research involved 28 individuals only and early loading implant treatment was applied. Patients did not have significant medical records, systemic diseases, or maxillofacial pathology. Increasing the sample size involving individuals with a concomitant pathology may have effect on the range of complaints and patients' attitude toward postoperative treatment. Despite these limitations, the study was aimed at understanding different patients' behavioral models concerning dental treatment and comprehension of prescribed recommendation as important factors of successful implant treatment using early loading protocol.

\section{Conclusions}

According to the results within the limitations of this study, it can be concluded that the compliance with postoperative recommendations and prescriptions was highly dependent on the patients' psychological type.

Philosophical patients were motivated, prudent, and ready for the treatment, $62.5-87.5 \%$ of patients completely followed treatment recommendations; Exacting-mind patients showed highly demanding attitude and accurateness $(66.7-100 \%$ of recommendation compliance). Hysterical patients were emotionally unstable, showed high recall rate at the initial stage (up to $80 \%$ ) with dramatical decrease (20-0\%) at later periods of observations. Indifferent patients were disinterested, fully followed recommendations in $16.7 \%$ of cases only, required high level of dentists' attention and verbal motivation.

\section{Ethical Statement}

This research was conducted according to the WMA Declaration of Helsinki - "Ethical Principles for Medical Research Involving Human Subjects" and approved by the Ethics Committee of the Ivano-Frankivsk National Medical University (protocol No. 111/19 dated November 19, 2019).

\section{Informed Consent}

All patients gave informed consent to participate in the research, and their anonymity was preserved.

\section{Conflict of Interest}

The authors declare that no conflicts exist.

\section{Financial Disclosure}

The authors declared no financial support.

\section{References}

[1] Al-Rafee M. The epidemiology of edentulism and the associated factors: a literature review. Journal of Family Medicine and Primary Care [Internet]. 2020;9(4):1841-1843. Available from: https://doi.org/10.4103/jfmpc.jfmpc_1181_19
[2] Farzadmoghadam M, Mohammadi TM, Goudarzi R, Mohammadi M, Hasheminejad N. Is there a relationship between general and oral health-related quality of life in partially edentulous patients before and after implant treatment? A quasi-experimental study. Clinical Oral Implants Research [Internet]. 2020;31(6):557-564. Available from: https://doi.org/10.1111/clr.13593

[3] Shrestha B, Basnet BB, Adhikari G. A questionnaire study on the impact on oral health-related quality of life by conventional rehabilitation of edentulous patient. BDJ Open [Internet]. 2020;6(1):3. Available from: https://doi.org/10.1038/s41405-020-0029-5

[4] Lopes A, Maló P, de Araújo Nobre M, SánchezFernández E, Gravito I. The NobelGuide®All-on$4 \bowtie T r e a t m e n t$ Concept for rehabilitation of edentulous jaws: a retrospective report on the 7-years clinical and 5years radiographic outcomes. Clinical Implant Dentistry and Related Research [Internet]. 2016;19(2):233-244. Available from: https://doi.org/10.1111/cid.12456

[5] Bedrossian E, Bedrossian E. Treatment planning the edentulous mandible. Review of biomechanical and clinical considerations: an update. The International Journal of Oral \& Maxillofacial Implants [Internet]. 2019;34(3):e33-e41. Available from: https://doi.org/10.11607/jomi.7196

[6] Yarov YY. Assessment of the effectiveness of differential management of patients in the early postoperative period following the dental implant surgery. Zaporozhye Medical Journal [Internet]. 2018;20(6):832836. Available from: https://doi.org/10.14739/23101210.2018.6.146756

[7] Ilkiv M, Rozhko M, Hereliuk V. Impact of periodontal pocket depth on the efficiency of surgical treatment of generalized periodontitis. Galician Medical Journal [Internet]. 2020;27(3):E202032. Available from: https://doi.org/10.21802/gmj.2020.3.2

[8] Wolfart S. Implant prosthodontics: a patient-oriented strategy. Berlin: Quintessence Publishing Co. Ltd.; 2016. $728 \mathrm{p}$.

[9] De Angelis F, Papi P, Mencio F, Rosella D, Di Carlo S, Pompa G. Implant survival and success rates in patients with risk factors: results from a long-term retrospective study with a 10 to 18 years follow-up. Eur Rev Med Pharmacol Sci [Internet]. 2017;21(3):433-437. Available from: http://www.ncbi.nlm.nih.gov/pubmed/28239830

[10] Zadik Y, Abu-Tair J, Yarom N, Zaharia B, Elad S. The importance of a thorough medical and pharmacological history before dental implant placement. Australian Dental Journal [Internet]. 2012;57(3):388-392. Available from: https://doi.org/10.1111/j.1834-7819.2012.01717.x 
[11] Atieh MA, Morgaine KC, Duncan WJ. A qualitative analysis on participants' perspectives on oral implants. Clinical Oral Implants Research [Internet]. 2015;27(3):383-391. Available from: https://doi.org/10.1111/clr.12558

[12] Choudhary S, Kumar A, Arora H. Correlation of patient's mental attitude with age, sex, and educational level: a survey. European Journal of Dentistry [Internet]. 2016;10(01):023-028. Available from: https://doi.org/10.4103/1305-7456.175688

[13] Goodacre CJ, Naylor WP. Evolution of the temperament theory and mental attitude in complete denture prosthodontics: from Hippocrates to M.M. House. Journal of Prosthodontics [Internet]. 2020;29(7):594-598. Available from: https://doi.org/10.1111/jopr.13215

[14] Matsumura Y, Hinode D, Fukui M, Yoshioka M, Asakuma H, Takii H. Effectiveness of an oral care tablet containing kiwifruit powder in reducing oral bacteria in tongue coating: a crossover trial. Clinical and Experimental Dental Research [Internet]. 2020;6(2):197-206. Available from: https://doi.org/10.1002/cre2.262

[15] Lundgren T, Mobilia A, Hallström H, Egelberg J. Evaluation of tongue coating indices. Oral Diseases [Internet]. 2007;13(2):177-180. Available from: https://doi.org/10.1111/j.1601-0825.2006.01261.x

[16] Jordan AR, Stark H, Nitschke I, Micheelis W, Schwendicke F. Epidemiological trends, predictive factors, and projection of tooth loss in Germany 1997-2030: part I. Missing teeth in adults and seniors. Clinical Oral Investigations [Internet]. 2020 Nov 21;25(1):67-76. Available from: https://doi.org/10.1007/s00784-020-03266-9

[17] Wolfart S, Gehrt M, Groß D. Management prothetischer komplikationen in der implantatprothetik. Implantologie. 2011;(4):395-408.

[18] Krupnyk AS. Incidence of small bounded edentulous spaces in children and adolescents living in Lviv. Galician Medical Journal [Internet]. 2017;24(1):E201712. Available from: https://doi.org/10.21802/gmj.2017.1.2

[19] Gamer S, Tuch R, Garcia LT. M. M. House mental classification revisited: intersection of particular patient types and particular dentist's needs. The Journal of Prosthetic Dentistry [Internet]. 2003;89(3):297-302. Available from: https://doi.org/10.1067/mpr.2003.49

[20] Monje A, Pérez A, Vera M, Nart J, Catena A, Petrova D. Comprehension and recall of information about factors associated to peri-implantitis: a randomized controlled trial. Journal of Periodontology [Internet]. 2021. Available from: https://doi.org/10.1002/JPER.21-0018

[21] Prasad KD, Prasad AD. Applied aspects of behavioural sciences in prosthodontics. Journal of Evolution of Medical and Dental Sciences [Internet]. 2020;9(15):1305-1309. Available from: https://doi.org/10.14260/jemds/2020/283

[22] Sangappa SB. Patient satisfaction in prosthodontic treatment: multidimensional paradigm. The Journal of Indian Prosthodontic Society [Internet]. 2011;12(1):21-26. Available from: https://doi.org/10.1007/s13191-0110106-5

[23] ELsyad MA, Maryod WH, Mostafa AZ. EEffect of implant position on clinical and radiographic outcomes of locator-retained mandibular overdentures: a 1-year prospective study. Journal of Prosthodontics [Internet]. 2018;28(2):e699-e704. Available from: https://doi.org/10.1111/jopr.12780

[24] Maniewicz S, Buser R, Duvernay E, Vazquez L, Loup A, Perneger T, et al. Short dental implants retaining twoimplant mandibular overdentures in very old, dependent patients: radiologic and clinical observation up to 5 years. The International Journal of Oral \& Maxillofacial Implants [Internet]. 2017;32(2):415-422. Available from: https://doi.org/10.11607/jomi.5361

[25] Alsabeeha NHM, Payne AGT, De Silva RK, Thomson WM. Mandibular single-implant overdentures: preliminary results of a randomised-control trial on early loading with different implant diameters and attachment systems. Clinical Oral Implants Research [Internet]. 2010;22(3):330-337. Available from: https://doi.org/10.1111/j.1600-0501.2010.02004.x

[26] Smith AJE. Series: Communication in the dental practice. Building a good dentist-patient relationship with communication. Nederlands Tijdschrift voor Tandheelkunde [Internet]. 2019;126(01):37-44. Available from: https://doi.org/10.5177/ntvt.2019.01.18212

Received: 2020-10-24

Revised: $2021-03-26$

Accepted: 2021-04-20 


\section{Appendix 1}

\section{Pharmacological protocol for the postoperative period}

Systemic agents:

- An antibiotic of broad-spectrum penicillin group with a beta-lactamase inhibitor for 5 days.

- Symbiotic for 14 days.

- Non-steroidal anti-inflammatory drug with predominant inhibitory activity against the enzyme COX-2. Signature: in case of acute pain.

Topical agents:

- Antibacterial tooth-wash $0.12 \%$ Chlorhexidine-denta. Signature: rinsing the mouth after each meal throughout the postoperative period.

- Solcoseryl dental adhesive paste. Signature: apply to the tissues of the surgical area after each rinsing.

\section{Appendix 2}

\section{Recommendations for the postoperative period}

- Ice application (wrapped in a towel) for 15 minutes every hour on the first day after surgery.

- Avoid physical activity.

- Not to eat or drink for two hours after surgery.

- Not to eat hot food for 2 days.

- Abstention from smoking and alcohol consumption.

- Compliance with the pharmacological protocol according to the medication administration record.

\section{Appendix 3}

Questionnaire regarding complaints, compliance with pharmacological protocol and recommendations

Dear !

We ask you to answer a few questions in order to obtain information on the course of your postoperative period and to monitor compliance with appointments and recommendations!

\begin{tabular}{l|l|l|l}
\hline \multicolumn{2}{l|}{ Date of surgical stage of implant treatment: } & \multicolumn{3}{l}{} \\
\cline { 3 - 4 } Day & I. Your complaints & $\begin{array}{l}\text { II. Do you comply with the pre- } \\
\text { scribed pharmacological proto- } \\
\text { col? }\end{array}$ & $\begin{array}{l}\text { III. Do you comply with pre- } \\
\text { scribed recommendations? }\end{array}$ \\
\hline $2^{\text {nd }}$ day & & & \\
\hline $4^{\text {th }}$ day & & & \\
\hline $8^{\text {th }}-10^{\text {th }}$ days & & & \\
\hline $12^{\text {th }}-14^{\text {th }}$ days & & & \\
\hline
\end{tabular}

- Indicate all, with no exception, complaints that you have in paragraph I.

- Indicate the level of compliance with the prescribed pharmacological protocol (fully comply with; partially comply with (indicate what is not complied with); do not comply with) in paragraph II.

- Indicate the level of compliance with the prescribed recommendations (fully comply with; partially comply with (indicate what is not complied with); do not comply with) in paragraph III. 